\title{
Effects of Overdispersion on Testing for Serial Dependence in the Time Series of Counts Data
}

\author{
Hee-Young Kim ${ }^{1, a}$, Yousung Park ${ }^{b}$ \\ ${ }^{a}$ Institute of Economics, Korea University; ${ }^{b}$ Department of Statistics, Korea University
}

\begin{abstract}
To test for the serial dependence in time series of counts data, Jung and Tremayne (2003) evaluated the size and power of several tests under the class of INARMA models based on binomial thinning operations for Poisson marginal distributions. The overdispersion phenomenon(i.e., a variance greater than the expectation) is common in the real world. Overdispersed count data can be modeled by using alternative thinning operations such as random coefficient thinning, iterated thinning, and quasi-binomial thinning. Such thinning operations can lead to time series models of counts with negative binomial or generalized Poisson marginal distributions. This paper examines whether the test statistics used by Jung and Tremayne (2003) on serial dependence in time series of counts data are affected by overdispersion.
\end{abstract}

Keywords: Overdispersion, negative binomial, generalized Poisson, time series of counts data, serial dependence.

\section{Introduction}

Time series of counts are commonly observed in a wide range of real-world applications and there is increased research interest in their analysis for time series modeling. The starting point is the test for serial dependence in data. Recently, Jung and Tremayne (2003) proposed several tests for serial correlation and evaluated the size and power of those tests against integer-valued autoregressive moving average(INARMA) models with Poisson marginal distributions. INARMA models, introduced by McKenzie (1985), use binomial thinning instead of scalar multiplication, and their correlation structure is similar to that of Gaussian ARMA models. In particular, the INAR(1) model using a binomial thinning operator can be applied to Poisson, negative binomial(NB), and generalized Poisson(GP) marginal distributions.

Our concern here is the behavior of the test statistics in Jung and Tremayne (2003) under the overdispersed INAR(1) model. Because the INAR(1) model is stationary, its innovation distribution is determined by its marginal distribution. The simplest Poisson INAR(1) model with a Poisson marginal distribution has Poisson innovations, which may be appropriate for modeling the time series of equi-dispersed small counts. Aside from the Poisson INAR(1) model, the explicit derivation of innovation distributions of the INAR(1) model is not easy. For the case of NB marginal, it can be derived in a quite complicated form, but it is not possible for the case of GP marginal, see Weiß (2008).

The INAR(1) model has undergone various generalizations to overcome this limitation, including the random-coefficient INAR(1) model with an NB marginal distribution (Zheng et al., 2007), the

\footnotetext{
${ }^{1}$ Corresponding author: Research Professor, Institute of Economics, Korea University, Anam-dong, Seongbuk-gu, Seoul 136-701, Korea. E-mail: starkim@korea.ac.kr
} 
iterated-thinning INAR(1) model with an NB distribution (Al-Osh and Aly, 1992), the quasi-binomial thinning INAR(1) model with a GP marginal distribution (Alzaid and Al-Osh, 1993).

As mentioned earlier, Jung and Tremayne (2003) used Monte Carlo techniques to evaluate the size and power properties of their proposed tests against INARMA models using binomial thinning operations; all of the models considered had Poisson marginal distributions.

The present paper examines the behavior of test statistics in Jung and Tremayne (2003) under various INAR(1) models. The rest of this paper proceeds as follows: Section 2 provides a brief overview of the INAR(1) models used for the comparison, including the random-coefficient INAR(1) model with an NB marginal distribution, the iterated-thinning INAR(1) model with an NB marginal distribution, and the quasi-binomial thinning INAR(1) model with a GP marginal distribution. Section 3 presents test statistics for the presence of serial dependence in the time series of counts. Section 4.1 provides the results of Monte Carlo simulation studies for the size properties of the test statistics under the null of i.i.d. Poisson, NB, and GP variables. Jung and Tremayne (2003) examined the size of the test statistics under the null of i.i.d. Poisson, NB variables for a few cases, but we conducted a large number of Monte Carlo studies focusing on overdispersion. Section 4.2 provides the empirical power of the tests against several INAR(1) models presented in Section 2. Section 5 concludes this study.

\section{Model Description}

This section briefly introduces NB and GP distributions, which can be marginal distributions of INAR(1) models, and summarizes several INAR(1) models with those distributions as marginal distributions.

The probability mass function(pmf) of the NB distribution with parameters $r$ and $p$, denoted by $\mathrm{NB}(r, p)$, is

$$
P(Y=y \mid r, p)=\frac{\Gamma(r+y)}{y ! \Gamma(r)} p^{r}(1-p)^{y}, \quad y=0,1, \ldots, r>0,0<p<1 .
$$

It is well known that the NB distribution is a mixed Poisson distribution with a gamma mixing distribution, so

$$
P(Y=y \mid \mu, \alpha)=\frac{\Gamma\left(\alpha^{-1}+y\right)}{\Gamma\left(\alpha^{-1}\right) \Gamma(y+1)}\left(\frac{\alpha^{-1}}{\alpha^{-1}+\mu}\right)^{\alpha^{-1}}\left(\frac{\mu}{\mu+\alpha^{-1}}\right)^{y}, \quad \mu>0, \alpha>0,
$$

with $E(y \mid \mu, \alpha)=\mu, \operatorname{Var}(y \mid \mu, \alpha)=\mu(1+\alpha \mu)$. Mixed Poisson distributions have overdispersion characteristics and have larger proportion of zeros than Poisson distributions. Therefore, the use of the mixed Poisson distribution can help us improve with respect to overdispersion and with respect to zero inflation (Nikoloulopoulos and Karlis, 2008).

Consul and Jain (1973) introduced the generalized Poisson(GP) distribution with a pmf, $\operatorname{GP}(\lambda, \theta)$, which is given by

$$
P(X=x \mid \theta, \lambda)= \begin{cases}\lambda(\lambda+\theta x)^{x-1} \frac{\exp (-\lambda-\theta x)}{x !}, & x=0,1, \ldots, \\ 0, & \text { for } x>m \text { when } \theta<0,\end{cases}
$$

where $\lambda>0, \max (-1,-\lambda / m)<\theta \leq 1, m>4$. The GP distribution reduces to the Poisson distribution when $\theta=0$. The mean and variance of the GP distribution are $E(X)=\lambda /(1-\theta)$ and $\operatorname{Var}(X)=$ $\lambda /(1-\theta)^{3}$. Therefore, the variance of the GP distribution can be greater or less than its mean depending 
Table 1: The relation between parameters and mean $\mu$, variance $\sigma^{2}$ of NB and GP distribution.

\begin{tabular}{ccccc}
\hline \hline Distribution & mean $\mu$ & variance $\sigma^{2}$ & \multicolumn{2}{c}{ Parameters } \\
\hline NB & $\frac{r(1-p)}{p}$ & $\frac{r(1-p)}{p^{2}}$ & $r=\frac{\mu^{2}}{\sigma^{2}-\mu}$ & $p=\frac{\mu}{\sigma^{2}}$ \\
GP & $\frac{\lambda}{1-\theta}$ & $\frac{\lambda}{(1-\theta)^{3}}$ & $\lambda=\mu \sqrt{\frac{\mu}{\sigma^{2}}}$ & $\theta=1-\sqrt{\frac{\mu}{\sigma^{2}}}$ \\
\hline \hline
\end{tabular}

on whether $\theta$ is positive or negative. Recently, Joe and Zhu (2005) proved that GP is a mixed Poisson distribution when $\theta>0$.

Table 1 shows the mean $\mu$ and variance $\sigma^{2}$ of these two distributions as functions of their parameters and vice versa. Refer to Nikoloulopoulos and Karlis (2008) and Joe and Zhu (2005) for more information on the properties of NB and GP distributions.

\subsection{The INAR(1) model with a Poisson marginal distribution}

When the observed time series are a low frequency count, the autoregressive moving average(ARMA) models cannot be applied to an integer-valued case. Because multiplying an integer by a real number generally does not give an integer value, there is a need for an operation with similar properties that can replace the scalar multiplication in ARMA models. One option is the thinning operation that is a probabilistic operation that can be applied to non-negative integer-valued random variables. For a given $X=x$ with a range $\mathbb{N}_{0}, x$ can be the number of some objects produced, the number of some events. Thinning operations select some objects of $x$ due to a certain random selection.

The Binomial Thinning Operation by Steutel and van Harn (1979) is the most popular thinning operation. Let $X$ be a non-negative integer-valued random variable. For $\rho \in[0,1]$, define the random variable

$$
\rho \circ X \equiv \sum_{i=1}^{X} Y_{i},
$$

where $\left\{Y_{i}\right\}$ are i.i.d. Bernoulli random variables independent of $X$ such that $P\left(Y_{i}=1\right)=1-P\left(Y_{i}=\right.$ $0)=\rho$.

The INAR(1) model introduced by McKenzie (1985) and Al-Osh and Alzaid (1987) is

$$
X_{t}=\rho \circ X_{t-1}+\varepsilon_{t},
$$

where all thinning operations are performed independently of each other and of $\left\{\varepsilon_{t}\right\}$ and the thinning operations at $t$ and $\left\{\varepsilon_{t}\right\}$ are independent of $\left\{X_{s}, s<t\right\}$.

In particular, if $\left\{\varepsilon_{t}\right\}$ is i.i.d. Poisson $(\lambda)$ and $X_{0}$ is Poisson $(\lambda /(1-\rho))$, then the marginal distribution $\left\{X_{t}\right\}$ is Poisson $(\lambda /(1-\rho))$ and is called the Poisson INAR(1) model. The Poisson INAR(1) model is the simplest INAR(1) model and is appropriate for modeling the time series of equi-dispersed counts.

\subsection{The INAR(1) model with a negative binomial marginal distribution(NB-INAR(1))}

Weiß (2008) showed that in the case of NB marginals, the probability mass function of $\varepsilon_{t}$ in Equation (2.2) is

$$
P\left(\varepsilon_{t}=k\right)= \begin{cases}(1-(1-p)(1-\rho))^{n}, & k=0, \\
\sum_{m=1}^{n}\left(\begin{array}{l}
n \\
m
\end{array}\right) \rho^{n-m}(1-\rho)^{m}\left(\begin{array}{c}
m+k-1 \\
k
\end{array}\right)(1-p)^{k} p^{m}, & k>0 .\end{cases}
$$


Because Equation (2.3) is complex, the NB-INAR(1) model may not be practical for real-world applications, and it is a possible reason why previous studies have considered other thinning operations.

\subsection{The random-coefficient INAR(1) model with a negative binomial marginal distribution(NB-RCINAR(1))}

In some situations, the parameter $\rho$ in Equation (2.2) may vary with time and be random. For example, let $X_{t}$ denote the number of terminally ill patients in the $t$ th month. Then we can assume that $X_{t}$ temporarily satisfies the INAR(1) model, where $\rho \circ X_{t-1}$ is the number of surviving patients from the previous month and $\varepsilon_{t}$ indicates newly admitted patients in the current month. However, the survival rate $\rho$ can vary randomly over time because of various environmental factors such as the quality of health care and the health of the patient. Joe (1996) and Zheng et al. (2007) extended the binomial thinning in Equation (2.1) to Random-Coefficient Thinning by replacing the fixed $\rho$ with a random parameter $\rho_{t}$ and considering $\rho_{t}$ as the realizations of i.i.d. random variables taking values in the interval $[0,1)$.

A process $\left\{X_{t}\right\}$ is an $\operatorname{RCINAR(1)~model~with~NB~marginals~} \mathrm{NB}(n, p)$ if

$$
X_{t}=\beta_{n, t} \circ X_{t-1}+\varepsilon_{t}, \quad X_{0} \sim \mathrm{NB}(n, p),
$$

where $\left\{\varepsilon_{t}\right\}$ is i.i.d. $\mathrm{NB}(n(1-\rho), p) ;\left\{\beta_{n, t}\right\}$ is i.i.d. $\operatorname{Beta}(n \rho, n(1-\rho))$, that is, $E\left(\beta_{n, t}\right)=\rho, \operatorname{Var}\left(\beta_{n, t}\right)=$ $\rho(1-\rho) /(n+1)$ independent of $\left\{\varepsilon_{t}\right\}$; and each $\beta_{n, t}$ is independent of $\left\{X_{s}, s<t\right\}$.

\subsection{The iterated-thinning INAR(1) model with a negative binomial marginal distribution(NB-IINAR(1))}

Al-Osh and Aly (1992) generalized binomial thinning operations to iterated thinning operations as follows:

$$
\rho *_{\alpha} X \equiv \sum_{i=1}^{(\alpha \rho) \circ X} Y_{i}, \quad 0<\alpha, \rho<1
$$

where $Y_{i}$ are i.i.d. $\mathrm{NB}(1, \alpha /(1+\alpha))$, which are independent of both $X$ and the thinning $(\alpha \rho) \circ X$. Here $(\alpha \rho) \circ X$ selects $(\alpha \rho) \circ X$ among $X$ individuals, and then each selected individuals give a random response $Y$ independently of other selected individuals.

Let $0<\alpha, \rho<1$ and $\left\{\varepsilon_{t}\right\}$ be an i.i.d. $\mathrm{NB}(n, \alpha /(1+\alpha))$. Then, assuming that all thinning operations are performed independently; independent $\left\{\varepsilon_{t}\right\}$ and that the thinnings at time $t$ and $\varepsilon_{t}$ are independent of $\left\{X_{s}, s<t\right\}$, the process $\left\{X_{t}\right\}$ defined by the recursion

$$
X_{t}=\rho *_{\alpha} X_{t-1}+\varepsilon_{t}, \quad t \geq 1, X_{0} \sim \mathrm{NB}\left(n, \frac{\alpha(1-\rho)}{1+\alpha(1-\rho)}\right),
$$

has a marginal distribution $\operatorname{NB}(n, \alpha(1-\rho) /(1+\alpha(1-\rho)))$.

\subsection{The quasi-binomial thinning INAR(1) model with a generalized Poisson marginal distribution(GP-QINAR(1))}

The quasi-binomial thinning INAR(1) model proposed by Alzaid and Al-Osh (1993) is defined as

$$
X_{t}=\rho_{\theta, \lambda} \circ X_{t-1}+\varepsilon_{t},
$$


Table 2: The comparison of several models-marginal mean and marginal variance

\begin{tabular}{cccc}
\hline \hline Model & $E\left(X_{t}\right)$ & \multicolumn{1}{c}{$\operatorname{Var}\left(X_{t}\right)$} & $E\left(X_{t} \mid X_{t-1}\right)$ \\
\hline NB-INAR(1) & $\frac{\mu_{\varepsilon}}{1-\rho}$ & $\frac{\rho \mu_{\varepsilon}+\sigma_{\varepsilon}^{2}}{1-\rho^{2}}$ & $\rho X_{t-1}+\mu_{\varepsilon}$ \\
NB-RCINAR(1) & $\frac{n(1-p)}{p}$ & $\frac{n(1-p)}{p^{2}}$ & $\rho X_{t-1}+\frac{n(1-\rho)(1-p)}{p}$ \\
NB-IINAR(1) & $\frac{n}{\alpha(1-\rho)}$ & $\frac{n(1+\alpha(1-\rho))}{(\alpha(1-\rho))^{2}}$ & $\rho X_{t-1}+\frac{n}{\alpha}$ \\
GP-QINAR(1) & $\frac{\lambda}{1-\theta}$ & $\frac{\lambda}{(1-\theta)^{3}}$ & $\rho X_{t-1}+\frac{\lambda(1-\rho)}{1-\theta}$ \\
\hline \hline
\end{tabular}

where $\varepsilon_{t}$ are i.i.d. $\operatorname{GP}((1-\rho) \lambda, \theta)$ and $X_{0} \sim \operatorname{GP}(\lambda, \theta)$. The $\rho_{\theta, \lambda} \circ X_{t-1}$ in Equation (2.7) is called the quasi-binomial thinning operation and is defined as follows. If $X_{t-1}=x$ is given, then $\rho_{\theta, \lambda} \circ X_{t-1}$ has a quasi-binomial distribution with parameters $(x, \rho, \theta / \lambda)$, that is,

$$
P\left(\rho_{\theta, \lambda} \circ X_{t-1}=i \mid X_{t-1}=x\right)=\frac{\rho(1-\rho)\left(\begin{array}{c}
x \\
i
\end{array}\right)\left(\rho+i \frac{\theta}{\lambda}\right)^{i-1}\left(1-\rho+(x-i) \frac{\theta}{\lambda}\right)^{x-i-1}}{\left(1+x \frac{\theta}{\lambda}\right)^{x-1}}, \quad i=0,1, \ldots, x .
$$

If the thinnings in Equation (2.7) are performed independently of each other and the thinning at time $t$ and $\varepsilon_{t}$ are independent of $\left\{X_{s}, s<t\right\}$, then $\left\{X_{t}\right\}$ is a stationary process with a marginal distribution $\operatorname{GP}(\lambda, \theta)$.

Table 2 summarizes the mean, variance, and conditional mean of the abovementioned models. Note that all models have the same autocorrelation function, $\operatorname{Corr}\left(X_{t}, X_{t-k}\right)=\rho^{k}$, which is similar to that of the AR(1) process except that it is nonnegative. For a thorough review of thinning operations, the reader is referred to Weiß (2008).

\section{Test Statistics for the Presence of Serial Independence}

In this section we briefly enumerate the test statistics for the presence of serial dependence in count data $\left\{x_{1}, \ldots, x_{T}\right\}$ (for more information, see Jung and Tremayne (2003)). We also consider the Ljung and Box (1978) statistic, which is commonly available in statistical software package.

The Wald-Wolfowitz test (1940) test, also known as the Runs test for randomness, is used to test the hypothesis that a series of counts is random. The first step in the runs test is the centering of data about their mean, and the statistic $Z$ is defined as

$$
Z=\frac{R-E(R)}{\sqrt{\operatorname{Var}(R)}},
$$

where $R$ is the number of runs, $E(R)=(2 n m /(n+m))+1$ and $\operatorname{Var}(R)=(2 n m(2 n m-n-m)) /((n+$ $\left.m)^{2}(n+m-1)\right)$ in which $n$ is the number of positive runs and $m$ is the number of negative runs. The statistic $Z$ is asymptotically distributed as $N(0,1)$ under the independence of the observable series of counts.

Freeland (1998) proposed the score statistic $S$ defined as

$$
S=\frac{\sqrt{T} \sum_{t=2}^{T}\left(X_{t-1}-\bar{X}\right)\left(X_{t}-\bar{X}\right)}{\sum_{t=1}^{T} X_{t}},
$$


where $\bar{X}$ is the sample mean. Under the null of i.i.d. Poisson variables, $S \stackrel{d}{\rightarrow} N(0,1)$. A modified $S^{*}$ is defined as

$$
S^{*}=\frac{\sqrt{T} \sum_{t=2}^{T}\left(X_{t-1}-\bar{X}\right)\left(X_{t}-\bar{X}\right)}{\sum_{t=1}^{T}\left(X_{t}-\bar{X}\right)^{2}} .
$$

Note that $S^{*}$ differs from $S$ with respect to the denominator term, which makes it preferable if overdispersion is present. Also $S^{*} \stackrel{d}{\rightarrow} N(0,1)$ under hypothesis of i.i.d. Poisson random variables.

Under the null hypothesis of i.i.d. random variables, the adapted version of the statistic given in Venkataraman (1982) is

$$
Q_{a c f}(k)=\sum_{i=1}^{k} \frac{\hat{\rho}_{i+1}^{2}\left[\sum_{t=1}^{T}\left(X_{t}-\bar{X}\right)^{2}\right]^{2}}{\sum_{t=i+2}^{T}\left(X_{t}-\bar{X}\right)^{2}\left(X_{t-i-1}-\bar{X}\right)^{2}},
$$

where

$$
\hat{\rho}_{i}=\frac{\sum_{t=i+1}^{T}\left(X_{t}-\bar{X}\right)\left(X_{t-i}-\bar{X}\right)}{\sum_{t=1}^{T}\left(X_{t}-\bar{X}\right)^{2}}
$$

is the $i^{t h}$-order sample autocorrelation.

The second portmanteau-type test is an adapted version of Mills and Seneta (1989):

$$
Q_{p a c f}(k)=\sum_{i=1}^{k} \frac{\hat{\phi}_{i+1}^{2}\left[\sum_{t=1}^{T}\left(X_{t}-\bar{X}\right)^{2}\right]^{2}}{\sum_{t=i+2}^{T}\left(X_{t}-\bar{X}\right)^{2}\left(X_{t-i-1}-\bar{X}\right)^{2}},
$$

where $\hat{\phi}_{k}$ is the $k^{\text {th }}$-order sample partial autocorrelation. Under the null of i.i.d. variables, the limiting distribution of $Q_{a c f}(k)$ and $Q_{\text {pacf }}(k)$ is the $\chi^{2}$ distribution with the degree of freedom $k$.

Finally, we consider the Ljung and Box (1978) statistic. Although the statistic was not compared in Jung and Tremayne (2003), it is easily available in a statistical software package,

$$
\mathrm{LB}(k)=T(T+2) \sum_{i=1}^{k} \frac{\hat{\rho}_{i}^{2}}{T-i} .
$$

Under the null i.i.d. variables, $\operatorname{LB}(k) \stackrel{d}{\rightarrow} \chi^{2}(k)$.

We can note three facts.

(i) The $\operatorname{LB}(k)$ statistic is based on the $1^{s t}, \ldots, k^{\text {th }}$ sample autocorrelation functions, $Q_{a c f}(k)$ uses $2^{\text {nd }}, \ldots,(k+1)^{\text {th }}$ sample autocorrelation functions.

(ii) Because of $S^{*}=\sqrt{T} \hat{\rho}_{1}, S^{*} \stackrel{d}{\rightarrow} N(0,1)$ under the null of i.i.d. variables.

(iii) As mentioned, the limiting distribution of $Q_{a c f}(k)$ and $Q_{p a c f}(k)$ are the $\chi^{2}$ distribution with the degree of freedom $k$ under the null of i.i.d.variables (Mills and Seneta, 1989; Jung and Tremayne, 2006). 
Table 3: Rejection percentages under the independence of the tests under the i.i.d. Poisson assumption at the nominal 5\% significance level.

\begin{tabular}{|c|c|c|c|c|}
\hline$\lambda$ & Test & $T=50$ & $\begin{array}{l}T=100 \\
\end{array}$ & $T=500$ \\
\hline \multirow{12}{*}{5} & $\mathrm{Z}$ & 6.84 & 6.32 & 6.02 \\
\hline & $\mathrm{S}$ & 3.14 & 4.16 & 4.39 \\
\hline & $S^{*}$ & 3.28 & 4.18 & 4.44 \\
\hline & $Q_{a c f}(1)$ & 4.75 & 4.96 & 5.44 \\
\hline & $Q_{a c f}(5)$ & 5.07 & 4.74 & 5.03 \\
\hline & $Q_{a c f}(10)$ & 5.91 & 5.50 & 5.38 \\
\hline & $Q_{\text {pacf }}(1)$ & 5.62 & 5.44 & 5.39 \\
\hline & $Q_{p a c f}(5)$ & 5.12 & 5.00 & 4.98 \\
\hline & $Q_{p a c f}(10)$ & 5.70 & 5.13 & 5.12 \\
\hline & $L B(1)$ & 5.70 & 5.13 & 5.12 \\
\hline & $L B(5)$ & 5.70 & 4.91 & 5.22 \\
\hline & $L B(10)$ & 6.92 & 5.89 & 5.25 \\
\hline \multirow{12}{*}{10} & $\mathrm{Z}$ & 6.11 & 6.09 & 5.24 \\
\hline & $\mathrm{S}$ & 3.38 & 4.15 & 4.32 \\
\hline & $S^{*}$ & 3.28 & 4.12 & 4.27 \\
\hline & $Q_{a c f}(1)$ & 4.61 & 4.87 & 4.74 \\
\hline & $Q_{a c f}(5)$ & 4.97 & 5.02 & 4.81 \\
\hline & $Q_{a c f}(10)$ & 5.93 & 5.29 & 4.79 \\
\hline & $Q_{\text {pacf }}(1)$ & 5.41 & 5.10 & 4.79 \\
\hline & $Q_{\text {pacf }}(5)$ & 5.72 & 5.36 & 4.73 \\
\hline & $Q_{\text {pacf }}(10)$ & 6.14 & 5.12 & 4.87 \\
\hline & $L B(1)$ & 5.10 & 5.03 & 4.89 \\
\hline & $L B(5)$ & 5.41 & 4.87 & 5.05 \\
\hline & $L B(10)$ & 6.61 & 5.60 & 5.00 \\
\hline \multirow{12}{*}{15} & $\mathrm{Z}$ & 5.94 & 5.33 & 5.43 \\
\hline & $\mathrm{S}$ & 3.22 & 3.60 & 4.44 \\
\hline & $S^{*}$ & 3.17 & 3.76 & 4.49 \\
\hline & $Q_{a c f}(1)$ & 4.52 & 5.05 & 4.90 \\
\hline & $Q_{a c f}(5)$ & 5.03 & 5.19 & 4.94 \\
\hline & $Q_{a c f}(10)$ & 6.45 & 5.60 & 5.02 \\
\hline & $Q_{\text {pacf }}(1)$ & 5.02 & 5.35 & 4.86 \\
\hline & $Q_{p a c f}(5)$ & 5.32 & 5.51 & 5.06 \\
\hline & $Q_{\text {pacf }}(10)$ & 6.16 & 5.21 & 5.24 \\
\hline & $L B(1)$ & 4.82 & 4.66 & 4.75 \\
\hline & $L B(5)$ & 5.54 & 5.60 & 4.96 \\
\hline & $L B(10)$ & 6.95 & 5.91 & 5.35 \\
\hline
\end{tabular}

\section{Monte Carlo Study}

To examine the finite sample properties of tests, we conducted a Monte Carlo simulation. We will examine the empirical size and power of several test statistics in Section 3, that is, $Z, S, S^{*}, Q_{a c f}(k)$, $Q_{\text {pacf }}(k), \mathrm{LB}(k), k=1,5,10$. As mentioned in Section 3, Jung and Tremayne (2003) did not consider $\mathrm{LB}(1), \mathrm{LB}(5), \mathrm{LB}(10)$. All the simulations are conducted using programs written in SAS/IML procedure.

\subsection{The Size of Tests}

We generate a series $\left\{x_{1}, \ldots, x_{T}\right\}$ from the Poisson, NB and GP distributions with $E\left(X_{t}\right)=5,10,15$ and overdispersion (the variance-mean ratio) are set to $d=1.5,5,10$ in the case of the NB and GP distributions. The sample size are $T=50,100,500$ and the number of Monte Carlo replication is 10,000. Jung and Tremayne (2003) examined the size of statistics under the null of the i.i.d. $\operatorname{Poisson}(\lambda), \lambda=1,5$ and under the null of i.i.d. NB with $E\left(X_{t}\right)=1,5$ and $d=1.5,3$. 
Table 4: Rejection percentages under the independence of the tests under NB-overdispersion at the nominal 5\% significance level.

\begin{tabular}{|c|c|c|c|c|c|c|c|c|c|}
\hline \multirow{2}{*}{ Test } & \multicolumn{3}{|c|}{$d=1.5^{\dagger}$} & \multicolumn{3}{|c|}{$d=5$} & \multicolumn{3}{|c|}{$d=10$} \\
\hline & $T=50$ & $T=100$ & $T=500$ & $T=50$ & $T=100$ & $T=500$ & $T=50$ & $T=100$ & $T=500$ \\
\hline \multicolumn{10}{|l|}{$E\left(X_{t}\right)=5$} \\
\hline $\mathrm{Z}$ & 6.14 & 5.52 & 5.95 & 5.54 & 5.19 & 5.22 & 5.50 & 5.28 & 5.09 \\
\hline $\mathrm{S}$ & 9.26 & 11.14 & 12.53 & 28.57 & 31.09 & 34.69 & 32.19 & 34.38 & 39.28 \\
\hline$S^{*}$ & 3.03 & 4.13 & 4.59 & 3.93 & 4.06 & 4.40 & 4.00 & 4.37 & 5.14 \\
\hline$Q_{a c f}(1)$ & 5.17 & 5.00 & 4.76 & 5.09 & 5.54 & 5.65 & 5.21 & 6.54 & 6.08 \\
\hline$Q_{a c f}(5)$ & 5.11 & 4.89 & 5.09 & 4.62 & 5.80 & 5.31 & 5.19 & 7.46 & 7.90 \\
\hline$Q_{a c f}(10)$ & 5.95 & 5.27 & 5.12 & 5.57 & 6.39 & 5.65 & 6.28 & 8.37 & 8.95 \\
\hline$Q_{\text {pacf }}(1)$ & 6.24 & 5.61 & 4.85 & 6.71 & 6.40 & 5.78 & 7.87 & 8.15 & 6.54 \\
\hline$Q_{\text {pacf }}(5)$ & 6.13 & 5.30 & 5.07 & 6.98 & 7.28 & 5.60 & 10.41 & 10.37 & 8.46 \\
\hline$Q_{\text {pacf }}(10)$ & 6.62 & 5.32 & 5.26 & 9.08 & 7.94 & 5.94 & 14.14 & 12.54 & 9.65 \\
\hline$L B(1)$ & 4.65 & 5.31 & 4.66 & 4.28 & 4.22 & 4.38 & 3.58 & 3.88 & 4.47 \\
\hline$L B(5)$ & 5.07 & 5.18 & 4.94 & 4.31 & 4.46 & 4.40 & 3.84 & 4.44 & 4.55 \\
\hline$L B(10)$ & 6.34 & 5.79 & 5.29 & 4.67 & 4.93 & 4.83 & 4.27 & 4.32 & 4.74 \\
\hline \multicolumn{10}{|l|}{$E\left(X_{t}\right)=10$} \\
\hline $\mathrm{Z}$ & 5.72 & 5.07 & 5.48 & 5.22 & 4.90 & 5.06 & 5.53 & 4.88 & 5.21 \\
\hline S & 9.23 & 10.50 & 12.49 & 28.85 & 32.31 & 33.98 & 34.76 & 36.92 & 40.80 \\
\hline$S^{*}$ & 2.93 & 3.60 & 4.38 & 3.36 & 4.17 & 4.47 & 4.02 & 4.43 & 4.96 \\
\hline$Q_{a c f}(1)$ & 5.14 & 5.05 & 4.65 & 4.96 & 4.89 & 4.96 & 5.15 & 4.97 & 5.52 \\
\hline$Q_{a c f}(5)$ & 4.88 & 4.58 & 4.81 & 4.36 & 5.69 & 5.18 & 5.22 & 5.59 & 6.10 \\
\hline$Q_{a c f}(10)$ & 5.91 & 5.35 & 5.21 & 5.52 & 6.03 & 5.26 & 6.45 & 6.59 & 6.65 \\
\hline$Q_{\text {pacf }}(1)$ & 5.98 & 5.54 & 4.79 & 6.23 & 5.60 & 5.09 & 6.95 & 6.25 & 5.66 \\
\hline$Q_{\text {pacf }}(5)$ & 5.69 & 4.87 & 4.97 & 6.31 & 6.46 & 5.29 & 8.17 & 7.13 & 6.29 \\
\hline$Q_{p a c f}(10)$ & 5.80 & 5.04 & 5.13 & 7.22 & 6.31 & 5.28 & 10.37 & 8.09 & 6.85 \\
\hline$L B(1)$ & 4.97 & 4.70 & 5.07 & 4.63 & 5.06 & 4.88 & 4.28 & 4.37 & 4.88 \\
\hline$L B(5)$ & 5.44 & 4.86 & 4.92 & 4.70 & 5.45 & 4.81 & 4.48 & 4.30 & 5.17 \\
\hline$L B(10)$ & 6.66 & 5.38 & 5.01 & 5.52 & 5.49 & 5.12 & 5.18 & 4.92 & 5.26 \\
\hline \multicolumn{10}{|l|}{$E\left(X_{t}\right)=15$} \\
\hline $\mathrm{Z}$ & 6.06 & 4.93 & 5.40 & 5.75 & 4.82 & 5.02 & 4.82 & 5.15 & 4.95 \\
\hline $\mathrm{S}$ & 10.15 & 10.64 & 12.70 & 30.17 & 32.63 & 36.00 & 35.39 & 38.03 & 40.00 \\
\hline$S^{*}$ & 3.53 & 3.70 & 4.59 & 3.80 & 4.07 & 4.91 & 3.62 & 3.95 & 4.60 \\
\hline$Q_{a c f}(1)$ & 4.82 & 5.06 & 5.31 & 4.59 & 5.01 & 4.97 & 5.07 & 4.97 & 5.33 \\
\hline$Q_{a c f}(5)$ & 4.64 & 5.11 & 5.19 & 4.67 & 5.36 & 5.09 & 4.98 & 5.34 & 5.21 \\
\hline$Q_{a c f}(10)$ & 5.96 & 5.70 & 5.12 & 5.57 & 6.10 & 5.06 & 6.13 & 6.10 & 5.56 \\
\hline$Q_{\text {pacf }}(1)$ & 5.58 & 5.44 & 5.30 & 5.85 & 5.70 & 5.08 & 6.30 & 5.82 & 5.42 \\
\hline$Q_{p a c f}(5)$ & 5.59 & 5.53 & 5.17 & 5.72 & 5.75 & 5.35 & 6.73 & 6.25 & 5.68 \\
\hline$Q_{\text {pacf }}(10)$ & 5.71 & 5.66 & 5.18 & 6.42 & 6.67 & 5.19 & 8.17 & 7.41 & 5.83 \\
\hline$L B(1)$ & 5.54 & 5.00 & 5.12 & 4.72 & 5.09 & 5.04 & 4.28 & 4.54 & 4.81 \\
\hline$L B(5)$ & 5.33 & 5.12 & 5.42 & 4.70 & 5.01 & 5.15 & 4.79 & 4.67 & 5.16 \\
\hline$L B(10)$ & 6.66 & 5.78 & 5.19 & 5.64 & 5.69 & 5.21 & 5.45 & 5.31 & 5.03 \\
\hline
\end{tabular}

Notes: $d$ is overdispersion index defined by $\operatorname{Var}\left(X_{t}\right) / E\left(X_{t}\right)$.

Tables 3, 4 and 5 report the size performance under the three distributions at the $5 \%$ nominal size. The numbers in bold indicate values outside the $95 \%$ confidence interval, which was calculated by $0.05 \pm 1.96 \times \sqrt{0.05 \times(1-0.05) / 10000} \simeq(0.0457,0.0543)$. We now summarize the results shown in Tables 3, 4 and 5; some are the same as those in Jung and Tremayne (2003).

(1) Table 3 reports the rejection percentages of several tests under i.i.d. Poisson at the 5\% nominal size. The asymptotic theory of $Q_{a c f}(1)$ and $Q_{a c f}(5)$ supports the finite-sample behavior of these test statistics, but the over-rejection of $Q_{a c f}(10)$ continue until the size reached 100 . The $S$ and $S^{*}$ tests behave similarly; they tend to under-reject, and this tendency is weakened as the sample size increases. $Q_{\text {pacf }}(k)$ and $\operatorname{LB}(k), k=1,5,10$ tend to over-reject, and this tendency is weakened 
Table 5: Rejection percentages under the independence of the tests under GP-overdispersion at the nominal 5\% significance level.

\begin{tabular}{|c|c|c|c|c|c|c|c|c|c|}
\hline \multirow{2}{*}{ Test } & \multicolumn{3}{|c|}{$d=1.5^{\dagger}$} & \multicolumn{3}{|c|}{$d=5$} & \multicolumn{3}{|c|}{$d=10$} \\
\hline & $T=50$ & $T=100$ & $T=500$ & $T=50$ & $T=100$ & $T=500$ & $T=50$ & $T=100$ & $T=500$ \\
\hline \multicolumn{10}{|l|}{$E\left(X_{t}\right)=5$} \\
\hline $\mathrm{Z}$ & 6.34 & 5.81 & 5.52 & 5.77 & 4.77 & 5.39 & 5.27 & 5.00 & 5.12 \\
\hline S & 10.22 & 11.00 & 12.29 & 28.04 & 30.52 & 34.66 & 31.01 & 33.67 & 38.30 \\
\hline$S^{*}$ & 3.59 & 3.83 & 4.78 & 3.37 & 4.35 & 5.33 & 4.09 & 4.27 & 5.00 \\
\hline$Q_{a c f}(1)$ & 4.50 & 4.82 & 5.21 & 4.74 & 5.70 & 5.58 & 4.76 & 6.59 & 6.69 \\
\hline$Q_{a c f}(5)$ & 4.72 & 4.91 & 4.96 & 4.20 & 5.57 & 5.78 & 4.65 & 7.31 & 8.91 \\
\hline$Q_{a c f}(10)$ & 5.81 & 5.83 & 4.93 & 5.51 & 6.60 & 5.86 & 5.57 & 9.07 & 10.47 \\
\hline$Q_{\text {pacf }}(1)$ & 5.71 & 5.42 & 5.27 & 6.61 & 6.80 & 5.72 & 7.49 & 8.63 & 7.30 \\
\hline$Q_{\text {pacf }}(5)$ & 5.81 & 5.14 & 5.11 & 7.46 & 7.31 & 5.84 & 10.29 & 10.33 & 9.74 \\
\hline$Q_{\text {pacf }}(10)$ & 6.35 & 5.20 & 4.83 & 9.67 & 8.79 & 6.25 & 14.43 & 14.10 & 11.42 \\
\hline$L B(1)$ & 5.00 & 4.54 & 4.96 & 3.80 & 4.14 & 4.77 & 3.24 & 3.64 & 4.51 \\
\hline$L B(5)$ & 5.07 & 4.88 & 5.17 & 3.89 & 4.38 & 4.60 & 3.41 & 3.98 & 4.82 \\
\hline$L B(10)$ & 6.20 & 5.76 & 5.12 & 4.67 & 4.70 & 4.78 & 3.53 & 4.25 & 4.77 \\
\hline \multicolumn{10}{|l|}{$E\left(X_{t}\right)=10$} \\
\hline $\mathrm{Z}$ & 5.61 & 5.79 & 5.96 & 5.68 & 5.18 & 5.45 & 5.01 & 4.77 & 4.75 \\
\hline $\bar{S}$ & 9.42 & 11.09 & 13.17 & 29.40 & 32.45 & 34.43 & 33.32 & 35.74 & 40.01 \\
\hline$S^{*}$ & 3.26 & 3.62 & 4.98 & 3.67 & 4.24 & 4.77 & 3.89 & 4.32 & 4.76 \\
\hline$Q_{a c f}(1)$ & 4.97 & 5.29 & 5.01 & 4.47 & 5.11 & 5.01 & 4.85 & 5.56 & 5.52 \\
\hline$Q_{a c f}(5)$ & 4.97 & 4.87 & 4.99 & 4.61 & 5.25 & 5.46 & 4.05 & 5.48 & 6.27 \\
\hline$Q_{a c f}(10)$ & 5.92 & 5.66 & 5.26 & 5.55 & 5.82 & 5.62 & 5.18 & 6.79 & 6.88 \\
\hline$Q_{\text {pacf }}(1)$ & 5.83 & 5.66 & 5.04 & 5.71 & 6.01 & 5.23 & 6.86 & 6.64 & 5.88 \\
\hline$Q_{\text {pacf }}(5)$ & 5.58 & 4.99 & 5.30 & 6.32 & 6.08 & 5.64 & 6.88 & 7.09 & 6.48 \\
\hline$Q_{\text {pacf }}(10)$ & 5.70 & 5.17 & 5.03 & 8.01 & 6.72 & 5.75 & 10.06 & 9.34 & 7.38 \\
\hline$L B(1)$ & 5.44 & 5.18 & 5.16 & 4.70 & 4.87 & 4.91 & 3.72 & 4.37 & 4.88 \\
\hline$L B(5)$ & 5.39 & 4.97 & 5.49 & 4.80 & 4.58 & 4.93 & 3.65 & 4.33 & 5.15 \\
\hline$L B(10)$ & 6.51 & 5.80 & 5.14 & 5.74 & 5.31 & 5.27 & 3.99 & 4.65 & 4.88 \\
\hline \multicolumn{10}{|l|}{$E\left(X_{t}\right)=15$} \\
\hline $\mathrm{Z}$ & 5.93 & 5.20 & 5.24 & 4.97 & 4.96 & 4.84 & 5.32 & 4.99 & 5.08 \\
\hline S & 9.88 & 11.08 & 12.19 & 29.13 & 30.88 & 33.58 & 35.45 & 37.59 & 40.04 \\
\hline$S^{*}$ & 3.69 & 4.01 & 4.16 & 3.34 & 3.73 & 4.53 & 3.92 & 4.27 & 4.54 \\
\hline$Q_{a c f}(1)$ & 4.89 & 4.99 & 4.89 & 4.65 & 4.98 & 4.96 & 5.05 & 5.33 & 5.28 \\
\hline$Q_{a c f}(5)$ & 4.88 & 5.05 & 4.86 & 4.95 & 5.04 & 5.01 & 4.79 & 5.46 & 5.45 \\
\hline$Q_{a c f}(10)$ & 6.04 & 5.47 & 5.20 & 5.89 & 5.82 & 5.02 & 5.28 & 6.43 & 6.25 \\
\hline$Q_{\text {pacf }}(1)$ & 5.81 & 5.39 & 4.93 & 5.62 & 5.54 & 5.06 & 6.85 & 6.26 & 5.64 \\
\hline$Q_{\text {pacf }}(5)$ & 5.51 & 5.24 & 4.96 & 6.07 & 5.84 & 5.27 & 6.91 & 7.16 & 5.69 \\
\hline$Q_{\text {pacf }}(10)$ & 5.98 & 5.17 & 5.32 & 7.06 & 6.31 & 5.21 & 8.98 & 8.11 & 6.16 \\
\hline$L B(1)$ & 4.99 & 4.85 & 4.41 & 4.81 & 4.98 & 4.81 & 4.24 & 4.49 & 4.88 \\
\hline$L B(5)$ & 5.53 & 4.91 & 4.81 & 4.80 & 4.88 & 4.71 & 4.17 & 4.56 & 4.67 \\
\hline$L B(10)$ & 6.54 & 5.90 & 5.18 & 5.70 & 5.15 & 5.07 & 5.06 & 5.10 & 4.95 \\
\hline
\end{tabular}

considerably as the sample size increase.

(2) Table 4 shows the rejection percentages of the tests under the NB distribution at the $5 \%$ nominal size. Because the scaling of $S$ was based on the sample mean, overdispersion has a considerable influence on the rejection percentage of $S$. The rejection percentage of $S$ rapidly increases, and this problem worsens as the sample size increases. For example, when $d=10, T=500$ and $E\left(X_{t}\right)=5$, the percentage is 39.28. By contrast, $S^{*}$ gives under-rejection percentages, which are similar to those in Table 3. The $Q_{a c f}(k)$ and $Q_{p a c f}(k)$ tests are affected by overdispersion, but this problem is not as serious as that for $S$. In the case of $E\left(X_{t}\right)=5$, the highest rejection percentage is 14.14. Overdispersion influences $Q_{a c f}\left(k_{2}\right)$ considerably more than $Q_{a c f}\left(k_{1}\right)$ when $k_{1}<k_{2}$ and 


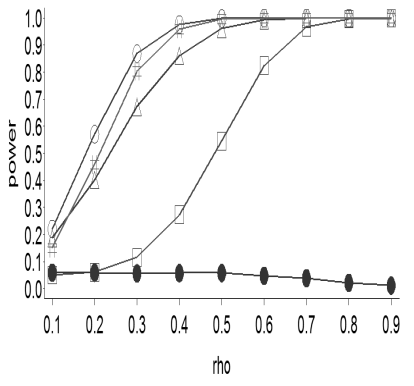

(a) $E\left(\varepsilon_{t}\right)=1$

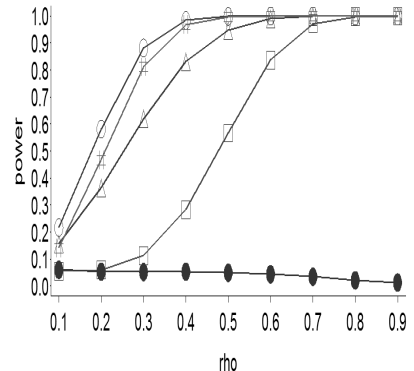

(b) $E\left(\varepsilon_{t}\right)=5$

Figure 1: Power curves for tests under Poisson INAR(1) alternative, based on 5\% level( $\triangle-Z, \bigcirc-S^{*}, \square-$

$$
\left.Q_{a c f}(1), \bullet-Q_{p a c f}(1), \sharp-L B(1)\right)
$$

influences $Q_{\text {pacf }}\left(k_{2}\right)$ more than $Q_{\text {pacf }}\left(k_{1}\right)$ when $k_{1}<k_{2}$. For the $Q_{a c f}(k)$ and $Q_{\text {pacf }}(k)$ tests, the over-rejection percentages decrease as the process mean increases. Unexpectedly, the LB test exhibits under-rejection for $d=1.5$ and over-rejection for $d=5,10$ when $E\left(X_{t}\right)=5$; this pattern disappears as the process mean increases to 15 .

(3) Table 5 shows the results under the GP distribution at the 5\% nominal size. The values and patterns are similar to those in Table 4. The results suggest that discriminating between the NB and GP distributions does not affect the behavior of the tests.

\subsection{Monte Carlo Power Properties}

We examine the finite-sample power properties of the tests in Section 3 through Monte Carlo simulations; the sample size is $T=100$ and the number of replications is 10,000 . Because the aim of this paper is to examine the behavior of the tests under various INAR(1) models discussed in Section 3, we analyze these models as alternative models: the random-coefficient INAR(1) model with an NB marginal distribution, the iterated-thinning INAR(1) model with an NB marginal distribution, and the quasi-binomial thinning INAR(1) model with a GP marginal distribution. Maintaining the same mean and overdispersion of the immigration process for the three models, we examine how the test statistics would differ against each alternative model. We consider the values $E\left(\varepsilon_{t}\right)=5,10$ and the values $d=\operatorname{Var}\left(\varepsilon_{t}\right) / E\left(X_{t}\right)=1.5,5$ and 10. Any departure from the null of i.i.d. variables is captured by the same parameter $\rho$ for all three models; the $\rho$ values are $0.1,0.2, \ldots, 0.9$.

We rule out the $S$ test because of its performance(Tables 4 and 5). In addition, as pointed out in Jung and Tremayne (2003), we do not report the results for $Q_{a c f}(k), Q_{p a c f}(k)$ and $\operatorname{LB}(k), k=$ 5,10 , because the power of tests is a declining function of the degree-of-freedom index for a given noncentrality parameter; this is confirmed by our experiments. Jung and Tremayne (2003) used size-adjusted critical values from their response surface analysis to evaluate the power of tests under the Poisson INAR(1), Poisson INMA(1) and Poisson INAR(2) models. The present study does not use a size-adjusted critical for the following reasons. As mentioned in Jung and Tremayne (2003), the influence of size distortions introduced by use of asymptotic critical values is restricted to small departures from the null. Obtaining size-adjusted critical values requires a response surface analysis for each distribution, which is beyond the scope of the present study. Finally, the results of using a size-unadjusted critical value might give useful information on the tests.

(1) Figure 1 presents the power curves for $Z, S^{*}, Q_{a c f}(1), Q_{\text {pacf }}(1)$ and $\operatorname{LB}(1)$ under the Poisson 


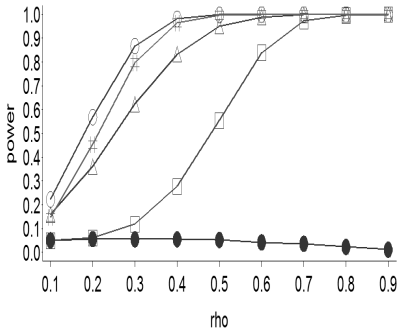

(a) $E\left(\varepsilon_{t}\right)=5, d=1.5$

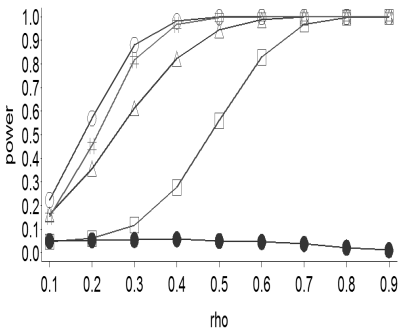

(d) $E\left(\varepsilon_{t}\right)=10, d=1.5$

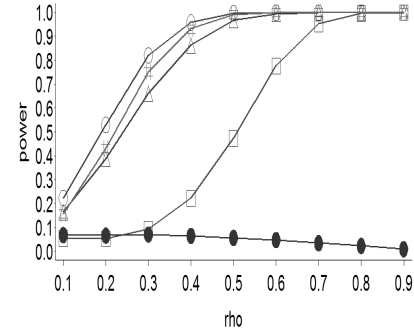

(b) $E\left(\varepsilon_{t}\right)=5, d=5$

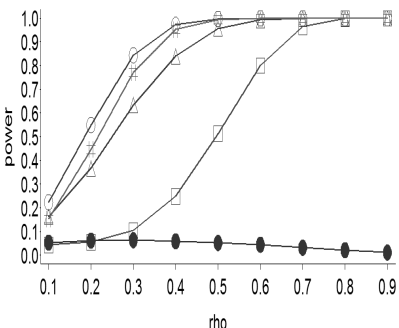

(e) $E\left(\varepsilon_{t}\right)=10, d=5$

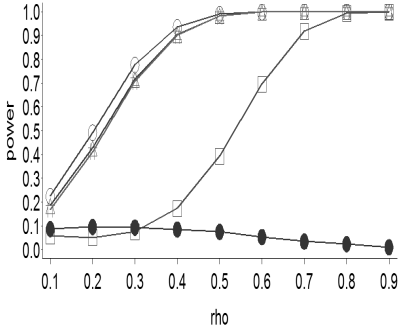

(c) $E\left(\varepsilon_{t}\right)=5, d=10$

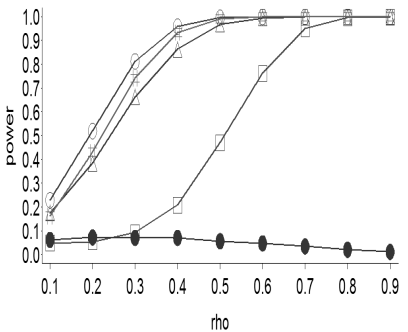

(f) $E\left(\varepsilon_{t}\right)=10, d=10$

Figure 2: Power curves for tests under NB-RCINAR(1) alternative, based on $5 \%$ level $\left(\triangle-Z, \bigcirc-S^{*}, \square-Q_{a c f}(1)\right.$, - $\left.-Q_{\text {pacf }}(1), \sharp-L B(1)\right)$

INAR(1) model in Equation (2.2). Noteworthy is that the present study considers the $\mathrm{LB}(1)$ test, which was not considered in Jung and Tremayne (2003). The power properties of LB(1) and $S^{*}$ are competitive across all the parameters considered in our study. The results indicate that the power of $Z$ is slightly worse than that of $S^{*}$ and $\operatorname{LB}(1)$ and that the power of $Q_{a c f}(1)$ is inferior to that of $Z$. The power of $Q_{\text {pacf }}(1)$ is nearly 0 . There is only a small difference between the power of the tests using size-unadjusted critical values in our experiment and that of those using size-adjusted critical values in Jung and Tremayne (2003); the maximum discrepancy is approximately 0.1 .

The changes of $E\left(\varepsilon_{t}\right)$ effects slightly the power of $Z$ among tests. Specifically, for each fixed $\rho$, the power of $Z$ decreases(range: $0 \sim 0.0535$ ) as $E\left(\varepsilon_{t}\right)$ increases.

(2) We now consider the second alternative model, the NB-RCINAR(1) model in Equation (2.4). We consider $E\left(\varepsilon_{t}\right)=5,10$ and $d=\operatorname{Var}\left(\varepsilon_{t}\right) / E\left(\varepsilon_{t}\right)=1.5,5,10$ and the correlation parameter $\rho=0.1,0.2, \ldots, 0.9$. The necessary parameters $n, p, \rho$ in Equation (2.4) to generate $x_{1}, \ldots, x_{T}$ are determined by the relation between the parameters and the mean/variance in Table 1 . The results shown in Figure 2 are summarized as follows. The order of power performance under the NB-RCINAR(1) alternative is the same as that under the Poisson INAR(1) alternative of $S^{*}>$ $L B(1)>Z>Q_{a c f}(1)>Q_{p a c f}(1)$. Although the effects of $d$ and $E\left(\varepsilon_{t}\right)$ are not immediately clear from Figure 2, for fixed $\rho$ and $E\left(\varepsilon_{t}\right)$, the power of $Z$ changes as $d$ varies. That is, an increase in $d$ leads to a slight increase in the power of $Z$. Indeed, the mean difference between $d=1.5$ and $d=5$ when $E\left(\varepsilon_{t}\right)=5$ was -0.0138 (range: $-0.0375 \sim 0$ ) and that between $d=1.5$ and $d=10$ when $E\left(\varepsilon_{t}\right)=5$ was -0.033 (range: $-0.0923 \sim 0$ ). This phenomenon persists in the case of $E\left(\varepsilon_{t}\right)=10$. Although these values are very small, the differences are always negative, which is inconsistent with the results under the Poisson INAR(1) model. As a result, Figure 2(c) shows 
Table 6: The mean of power difference of tests under alternative model

\begin{tabular}{|c|c|c|c|c|}
\hline \multirow{2}{*}{ Test } & \multirow{2}{*}{ Difference $^{\dagger}$} & \multicolumn{3}{|c|}{ Alternative model } \\
\hline & & NB-RCINAR(1) & NB-IINAR(1) & GP-QINAR(1) \\
\hline \multirow{6}{*}{$Z$} & diff12 & -0.0138 & 0.0187 & -0.0297 \\
\hline & $\operatorname{diff} 13$ & -0.0331 & 0.0422 & -0.0362 \\
\hline & $\operatorname{diff} 23$ & -0.0192 & 0.0153 & -0.0064 \\
\hline & $\operatorname{diff} 45$ & -0.0076 & 0.0041 & -0.0295 \\
\hline & $\operatorname{diff} 46$ & -0.0181 & 0.0163 & -0.0353 \\
\hline & $\operatorname{diff56}$ & -0.0106 & 0.0071 & -0.0058 \\
\hline \multirow{6}{*}{$S^{*}$} & diff12 & 0.0121 & 0.0274 & 0.0459 \\
\hline & $\operatorname{diff} 13$ & 0.0245 & 0.0584 & 0.0810 \\
\hline & $\operatorname{diff} 23$ & 0.0124 & 0.0177 & 0.0351 \\
\hline & $\operatorname{diff} 45$ & 0.0076 & 0.0133 & 0.0433 \\
\hline & $\operatorname{diff} 46$ & 0.0155 & 0.0329 & 0.0805 \\
\hline & diff56 & 0.0079 & 0.0110 & 0.0372 \\
\hline \multirow{6}{*}{$Q_{a c f}(1)$} & diff12 & 0.0273 & 0.0470 & 0.0832 \\
\hline & $\operatorname{diff} 13$ & 0.0570 & 0.0813 & 0.1359 \\
\hline & $\operatorname{diff} 23$ & 0.0296 & 0.0540 & 0.0527 \\
\hline & $\operatorname{diff} 45$ & 0.0145 & 0.0284 & 0.0824 \\
\hline & $\operatorname{diff} 46$ & 0.0307 & 0.0606 & 0.1335 \\
\hline & diff56 & 0.0162 & 0.0327 & 0.0511 \\
\hline \multirow{6}{*}{$L B(1)$} & diff12 & 0.0106 & 0.0278 & 0.0435 \\
\hline & $\operatorname{diff} 13$ & 0.0212 & 0.0609 & 0.0761 \\
\hline & $\operatorname{diff} 23$ & 0.0105 & 0.0193 & 0.0326 \\
\hline & $\operatorname{diff} 45$ & 0.0076 & 0.0148 & 0.0412 \\
\hline & $\operatorname{diff} 46$ & 0.0142 & 0.0344 & 0.0759 \\
\hline & diff56 & 0.0066 & 0.0111 & 0.0347 \\
\hline $\begin{array}{l}\text { Differ } \\
\text { diff12 } \\
\text { when } \\
\text { diff45 } \\
\text { when }\end{array}$ & $\begin{array}{l}\text { tes followi } \\
\text { ce between } \\
\text { diff } 23 \text { is di } \\
\text { ce between } \\
\text { diff56 is }\end{array}$ & $\begin{array}{l}\text { d } d=5 \text { when } E \\
\text { tween } d=5 \text { and } \\
\text { d } d=5 \text { when } E\end{array}$ & $\begin{array}{l}\text { diff } 13 \text { is differen } \\
\text { vhen } E\left(\varepsilon_{t}\right)=5 \text {. } \\
\text { diff46 is differe } \\
E\left(\varepsilon_{t}\right)=10\end{array}$ & $\begin{array}{l}l=1.5 \text { and } d=10 \\
d=1.5 \text { and } d=10\end{array}$ \\
\hline
\end{tabular}

an overlap between the power curves of LB and $Z$. Table 6 presents the mean differences in the power of tests for fixed $\rho$. The power of $S, Q_{a c f}(1)$ and $Q_{\mathrm{LB}}(1)$ tend to decrease as $d$ increases, whereas that of the other tests do not change as $d$ varies.

(3) The results under the alternative model NB-IINAR(1) are shown in Figure 3. The results indicate no substantial changes. The most powerful test is $S^{*}$, which is followed by $\operatorname{LB}(1)$ and $Z$, in that order. Consistent with the results under the Poisson INAR(1) and NB-RCINAR(1) alternatives, changes in $d$ has a minimal effect on $Z$. The mean difference between $d=1.5$ and $d=5$ when $E\left(\varepsilon_{t}\right)=5$ is 0.0187 (range: $0.0054 \sim 0.0315$ ) and that difference between $d=1.5$ and $d=10$ when $E\left(\varepsilon_{t}\right)=5$ is 0.0422 (range: $0.0164 \sim 0.0681$ ). Noteworthy is that as $d$ increases, its power decreases, which is inconsistent with the results under the NB-RCINAR(1) alternative.

(4) Figure 4 shows the results under the alternative model GP-QINAR(1). The notable findings are as follows. First, $d=1.5$ does not lead to changes in the order in the power of the tests, that is, $S^{*}>\mathrm{LB}>(1)>Z>Q_{a c f}(1)>Q_{\text {pacf }}(1)$. However, for $d=5,10$, the order of power performance is $Z>S^{*}>\mathrm{LB}(1)>Q_{a c f}(1)$, that is, the $Z$ test dominates the other tests, which is inconsistent with the results under the Poisson INAR(1), NB-RCINAR(1) and NB-IINAR(1) models. Second, for $d=5,10$, the power of $Q_{a c f}(1)$ is less than 0.3 until $\rho$ became 0.5 . Third, for fixed $\rho$ and $E\left(\varepsilon_{t}\right)$, the power of $Z$ increases as $d$ increases, but that of $S^{*}$ decrease. 


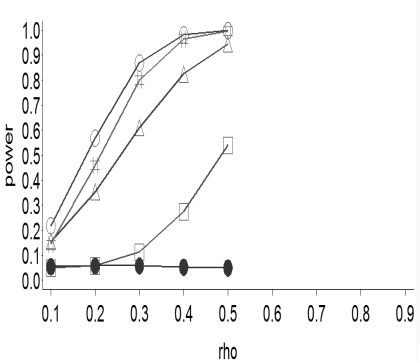

(a) $E\left(\varepsilon_{t}\right)=5, d=1.5$

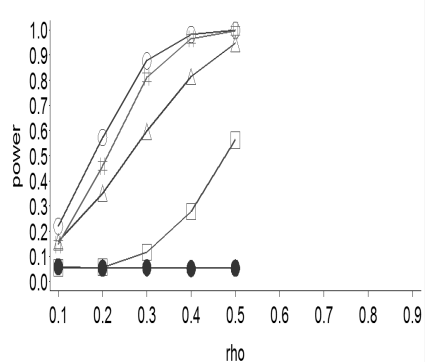

(d) $E\left(\varepsilon_{t}\right)=10, d=1.5$

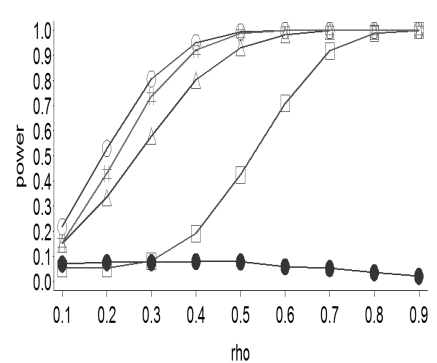

(b) $E\left(\varepsilon_{t}\right)=5, d=5$

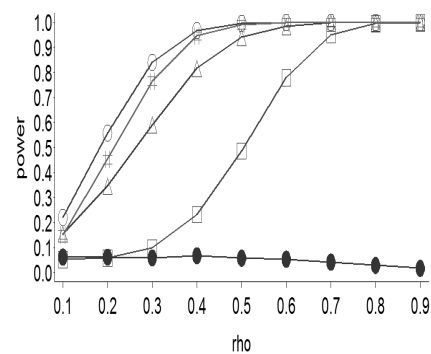

(e) $E\left(\varepsilon_{t}\right)=10, d=5$

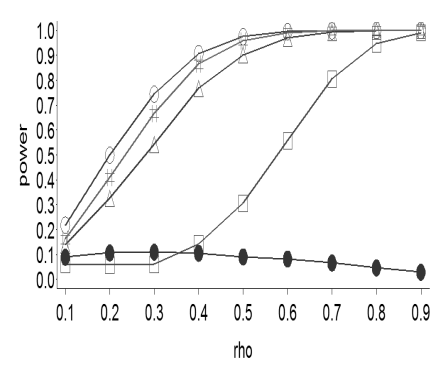

(c) $E\left(\varepsilon_{t}\right)=5, d=10$

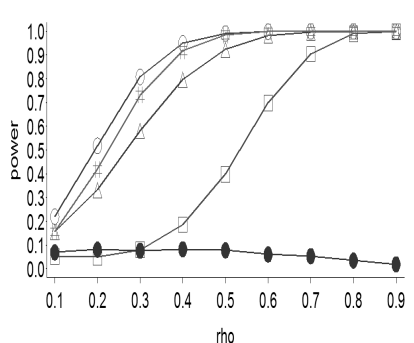

(f) $E\left(\varepsilon_{t}\right)=10, d=10$

Figure 3: Power curves for tests under NB-IINAR(1) alternative, based on $5 \%$ level( $\triangle-Z, \bigcirc-S^{*}, \square-Q_{a c f}(1)$, - $\left.Q_{\text {pacf }}(1), \sharp-L B(1)\right)$

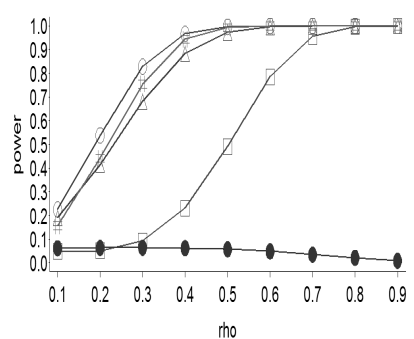

(a) $E\left(\varepsilon_{t}\right)=5, d=1.5$

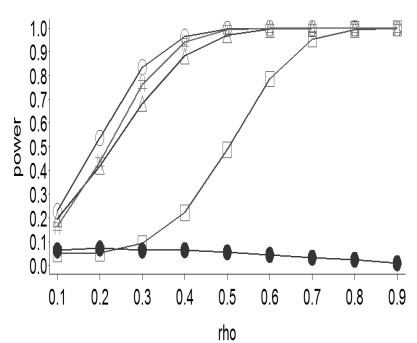

(d) $E\left(\varepsilon_{t}\right)=10, d=1.5$

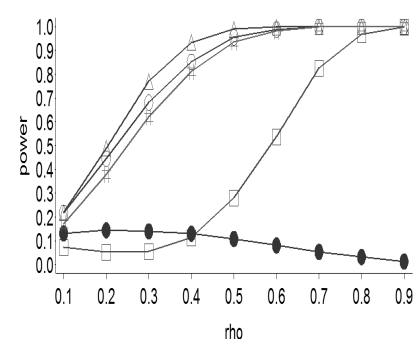

(b) $E\left(\varepsilon_{t}\right)=5, d=5$

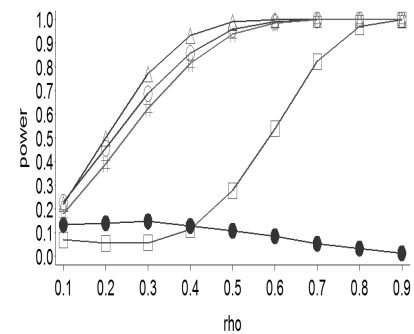

(e) $E\left(\varepsilon_{t}\right)=10, d=5$

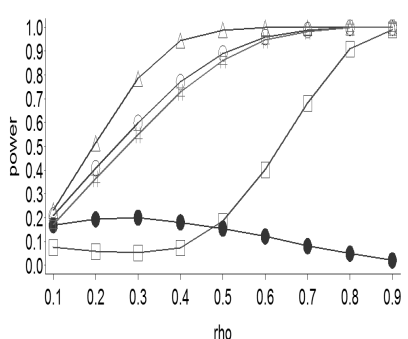

(c) $E\left(\varepsilon_{t}\right)=5, d=10$

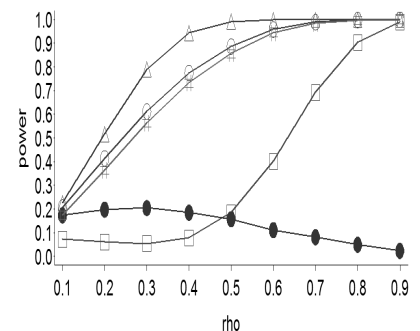

(f) $E\left(\varepsilon_{t}\right)=10, d=10$

Figure 4: Power curves for tests under GP-QINAR(1) alternative, based on $5 \%$ level( $\triangle-Z, \bigcirc-S^{*}, \square-Q_{a c f}(1)$, - $\left.-Q_{\text {pacf }}(1), \sharp-L B(1)\right)$ 
(5) Table 6 presents the mean differences in the power of the tests for fixed $\rho$; the third, fourth, and fifth column indicate the results under NB-RCINAR(1), NB-IINAR(1) and GP-INAR(1), respectively. The key observation is that for fixed $\rho, E\left(\varepsilon_{t}\right)$, the mean of the power difference in $S, Q_{a c f}(1)$ and $Q_{L B}(1)$ tend to decrease under all models as $d$ increase because the values for diff12, diff13, diff23, diff45, diff46 and diff56 are positive. The largest decrease is under the GP-QINAR(1) model because the values under the GP-QINAR(1) model are the largest.

\section{Conclusion}

In this paper, we revisited several tests for serial dependence in the times series of counts in Jung and Tremayne (2003) to evaluate their behavior under various overdispersed models. We compared the size and power of the runs test $Z$, the modified score test $S^{*}$, two portmanteau-type tests $Q_{a c f}(1)$ and $Q_{\text {pacf }}(1)$, and the Ljung and Box test LB(1), which was not considered in Jung and Tremayne (2003). The alternative models considered were the INAR(1) model with a Poisson marginal distribution, the random-coefficient INAR(1) model with a negative binomial marginal distribution(NB-INAR(1)), the iterative-thinning INAR(1) model with a negative binomial marginal distribution(NB-IINAR(1)), and the quasi-binomial thinning INAR(1) model with a generalized Poisson marginal distribution(GPQINAR(1)). The results of Monte Carlo simulations indicate that the overdispersion of the immigration process as well as the choice of the alternative model can affect the power performance of the tests. In general, the most powerful test was $S^{*}$, which was followed by $\operatorname{LB}(1), Z$ and $Q_{a c f}(1)$, in that order. However, the order was $Z>S^{*}>\mathrm{LB}(1)>Q_{a c f}(1)$ for some cases. The $Q_{\text {pacf }}(1)$ test had no power because we considered only first-order autoregressive models.

For fixed autocorrelation parameter $\rho$ and the mean of immigration process, as the overdispersion of the immigration process increase, the power of $S^{*}, Q_{a c f}(1)$ and $Q_{\mathrm{LB}}(1)$ tests tended to decrease and this tendency was the most under the GP-QINAR(1) model.

\section{References}

Al-Osh, M. A. and Aly, E. E. A. A. (1992). First order autoregressive time series with negative binomial and geometric marginals, Communications in Statistics-Theory and Methods, 21, 2483 2492.

Al-Osh, M. A. and Alzaid, A. A. (1987). First-order integer-valued autoregressive(INAR(1)) process, Journal of Time Series Analysis, 8, 261-275.

Alzaid, A. A. and Al-Osh, M. A. (1993). Some autoregressive moving average processes with generalized Poisson, Annals of the Institute of Statistical Mathematics, 45, 223-232.

Consul, P. C. and Jain, G. C. (1973). A generalized Poisson distribution, Technometrics, 15, 791-799.

Freeland, K. (1998). Statistical analysis of discrete time series with application to the analysis of workers compensation claims data, $\mathrm{PhD}$ thesis, University of British Columbia.

Joe, H. (1996). Time series models with univariate margins in the convolution-closed infinitely divisible class, Journal of Applied Probability, 33, 664-677.

Joe, H. and Zhu, R. (2005). Generalized Poisson distribution: The property of mixture of Poisson and comparison with negative binomial distribution, Biometrical Journal, 47, 219-229.

Jung, R. C. and Tremayne, A. R. (2003). Testing for serial dependence in time series models of counts, Journal of Time Series Analysis, 24, 65-84.

Jung, R. C. and Tremayne, A. R. (2006). Binomial thinning models for integer time series, Statistical Modelling, 6, 81-96. 
Ljung, G. M. and Box, G. E. P. (1978). On a measure of lack of fit in time series models, Biometrika, 65, 297-303.

McKenzie, E. (1985). Some simple models for discrete variate time series, Water Resour Bulletin, 21, 645-650.

Mills, T. M. and Seneta, E. (1989). Goodness-of-fit for a branching process with immigration using sample partial autocorrelations, Stochastic Processes and Their Applications, 33, 151-161.

Nikoloulopoulos, A. K. and Karlis, D. (2008). On modeling count data: A comparison of some wellknown discrete distributions, Journal of Statistical Computation and Simulation, 78, 437-457.

Steutel, F. W. and van Harn, K. (1979). Discrete analogues of self-decomposability and stability, The Annals of Probability, 7, 893-899.

Venkataraman, K. N. (1982). A time series approach to the study of the simple subcritical Galton Watson process with immigration, Advances in Applied Probability, 14, 1-20.

Wald, A. and Wolfowitz, J. (1940). On a test whether two samples are from the same population, Annals of Mathematical Statistics, 11, 147-162.

Weiß, C. H. (2008). Thinning operations for modeling time series of counts-a survey, Advances in Statistical Analysis, 92, 319-341.

Zheng, H., Basawa, I. V. and Datta, S. (2007). First-order random coefficient integer-valued autoregressive processes, Journal of Statistical Planning and Inference, 137, 212-229.

Received July 2010; Accepted August 2010 